AperTO - Archivio Istituzionale Open Access dell'Università di Torino

Impact of viral eradication with sofosbuvir-based therapy on the outcome of post-transplant hepatitis C with severe fibrosis

This is a pre print version of the following article:

Original Citation:

Availability:

This version is available http://hdl.handle.net/2318/1611544

since 2017-06-08T00:39:16Z

Published version:

DOI:10.1111/liv.13193

Terms of use:

Open Access

Anyone can freely access the full text of works made available as "Open Access". Works made available under a Creative Commons license can be used according to the terms and conditions of said license. Use of all other works requires consent of the right holder (author or publisher) if not exempted from copyright protection by the applicable law. 
Liver INTERNMTIONA

\section{Impact of Viral Eradication with Sofosbuvir-based Therapy on the Outcome of Post-Transplant Hepatitis C with Severe Fibrosis}

\begin{tabular}{|c|c|}
\hline Journal: & Liver International \\
\hline Manuscript ID & LIVint-16-00427.R2 \\
\hline Wiley - Manuscript type: & Original Articles \\
\hline Date Submitted by the Author: & $\mathrm{n} / \mathrm{a}$ \\
\hline Complete List of Authors: & $\begin{array}{l}\text { Martini, Silvia; AOU Città della Salute e della Scienza di Torino, } \\
\text { Gastrohepatology Unit } \\
\text { Sacco, Marco; AOU Città della Salute e della Scienza di Torino, } \\
\text { Gastrohepatology Unit } \\
\text { Strona, Silvia; AOU Città della Salute e della Scienza di Torino, } \\
\text { Gastrohepatology Unit } \\
\text { Arese, Daniele; AOU Città della Salute e della Scienza di Torino, } \\
\text { Gastrohepatology Unit } \\
\text { Tandoi, Francesco; AOU Città della Salute e della Scienza di Torino, Liver } \\
\text { Transplantation Center, General Surgery Unit } 2 \text { U } \\
\text { Dell Olio, Dominic; AOU Città della Salute e della Scienza di Torino, } \\
\text { Regional Transplantation Center, Piedmont } \\
\text { Stradella, Davide; AOU Città della Salute e della Scienza di Torino, } \\
\text { Gastrohepatology Unit } \\
\text { Cocchis, Donatella; AOU Città della Salute e della Scienza di Torino, Liver } \\
\text { Transplantation Center, General Surgery Unit } 2 \text { U } \\
\text { Mirabella, Stefano; AOU Città della Salute e della Scienza di Torino, Liver } \\
\text { Transplantation Center, General Surgery Unit } 2 \text { U } \\
\text { Rizza, Giorgia; AOU Città della Salute e della Scienza di Torino, Liver } \\
\text { Transplantation Center, General Surgery Unit } 2 \text { U } \\
\text { Magistroni, Paola; AOU Città della Salute e della Scienza di Torino, Regional } \\
\text { Transplantation Center, Piedmont } \\
\text { Moschini, Pamela; AOU Città della Salute e della Scienza di Torino, } \\
\text { Regional Transplantation Center, Piedmont } \\
\text { Ottobrelli, Antonio; AOU Città della Salute e della Scienza di Torino, } \\
\text { Gastrohepatology Unit } \\
\text { Amoroso, Antonio; AOU Città della Salute e della Scienza di Torino, } \\
\text { Regional Transplantation Center, Piedmont } \\
\text { Rizzetto, Mario; AOU Città della Salute e della Scienza di Torino, } \\
\text { Gastrohepatology Unit } \\
\text { Salizzoni, Mauro; AOU Città della Salute e della Scienza di Torino, Liver } \\
\text { Transplantation Center, General Surgery Unit } 2 \text { U } \\
\text { Saracco, Giorgio; AOU Città della Salute e della Scienza di Torino, } \\
\text { Gastrohepatology Unit } \\
\text { Romagnoli, Renato; AOU Città della Salute e della Scienza di Torino, Liver } \\
\text { Transplantation Center, General Surgery Unit } 2 U\end{array}$ \\
\hline Keywords: & Liver transplantation, HCV recurrence, Direct-acting antivirals, Non- \\
\hline
\end{tabular}




1
2
3
4
5
6
7
8
9
10
11
12
13
14
15
16
17
18
19
20
21
22
23
24
25
26
27
28
29
30
31
32
33
34
35
36
37
38
39
40
41
42
43
44
45
46
47
48
49
50
51
52
53
54
55
56
50

invasive fibrosis tests, Survival

SCHOLARONE ${ }^{\text {' }}$

Manuscripts

12

13

14

15

16

17

19

20

21

23

24

25

27

28

30

31

33

34

35

36

37

39

40

41

42

43

45

46

47

48

49

50

52

53

54

55

57

58

60 


\title{
Impact of Viral Eradication with Sofosbuvir-based Therapy on the Outcome of Post-Transplant Hepatitis C with Severe Fibrosis
}

\author{
Silvia Martini, ${ }^{1}$ Marco Sacco, ${ }^{1}$ Silvia Strona, ${ }^{1}$ Daniele Arese, ${ }^{1}$ Francesco Tandoi, ${ }^{2}$ \\ Dominic Dell Olio, ${ }^{3}$ Davide Stradella, ${ }^{1}$ Donatella Cocchis, ${ }^{2}$ Stefano Mirabella, ${ }^{2}$ \\ Giorgia Rizza, ${ }^{2}$ Paola Magistroni, ${ }^{3}$ Pamela Moschini, ${ }^{3}$ Antonio Ottobrelli, ${ }^{1}$ \\ Antonio Amoroso, ${ }^{3}$ Mario Rizzetto, ${ }^{1}$ Mauro Salizzoni, ${ }^{2}$ \\ Giorgio M. Saracco, ${ }^{1}$ Renato Romagnoli ${ }^{2}$ \\ ${ }^{1}$ Gastrohepatology Unit \\ ${ }^{2}$ Liver Transplantation Center, General Surgery Unit $2 U$ \\ ${ }^{3}$ Regional Transplantation Center, Piedmont \\ AOU Città della Salute e della Scienza di Torino, University of Turin, Italy
}

\section{Correspondence information}

Silvia Martini, MD

Gastrohepatology Unit, AOU Città della Salute e della Scienza di Torino

Corso Bramante, 88 - 10126 Turin - Italy

Phone: +39 011 6335928; fax: +390116334014

E-mail address: sivima21@hotmail.com

Electronic word count for main body of the manuscript: 4337.

Number of figures: 2 . Number of tables: 3 . 


\section{List of abbreviations in the order of appearance:}

HCV, hepatitis C virus; LT, liver transplantation; Peg-IFN, pegylated-interferon; SVR, sustained virological response; SVR12, sustained virological response at week 12 after the end of treatment; DAAs, direct-acting antiviral agents; LS, liver stiffness; kPa, kilopascal; EOT, end of therapy; IL28B, Interleukin 28B; HLA, human leukocyte antigen; AE, adverse event; AST, aspartate aminotransferase; APRI, AST to platelet ratio index; ALT, alanine aminotransferase; SVR24, sustained virological response at week 24 after the end of treatment; ROC, receiver operating characteristic; MELD, Model for End-stage Liver Disease; F, fibrosis; ITT, intention to treat.

\section{Conflict of interest}

MR is an Advisory Board member for AbbVie, BMS, Gilead, Janssen, MSD.

GMS is an Advisory Board member for AbbVie, Gilead and MSD.

$\mathrm{SM}$ is a Regional Advisory Board member for Gilead.

The other Authors who have taken part in this study declared no conflict of interest.

\section{Financial support}

This work was unsupported. 


\begin{abstract}
Background \& Aims. Several studies have shown that new direct-acting-antivirals (DAAs) maintain their efficacy in liver transplant (LT) recipients with severe HCV recurrence. We determined the clinical impact of Sofosbuvir/Ribavirin in LT through the changes in liver function and fibrosis state at 24 and 48 weeks after treatment.

Methods. Between 06/2014 and 07/2015, 126 patients (30 F3, 96 F4 Metavir stage) were enrolled to receive sofosbuvir+ribavirin (24 weeks, 118 patients) or

sofosbuvir+simeprevir+ribavirin (12 weeks, 8 patients); treatment was initiated at a median time of 4.3 years from LT. Median follow-up after therapy completion was 461 days. Results. All 30 F3 patients achieved a sustained virological response at week 24 after treatment (SVR24), and showed a distinct amelioration of the AST-to-platelet-ratio-index (APRI), FIB-4 and liver stiffness at elastography by week 24 post-therapy, which were maintained at week 48.

Of the 96 F4 cirrhotic patients, 72 (75\%) achieved SVR24 accompanied by significant improvement of liver function, which was maintained at week 48 (Child B-C 22\% baseline, $11 \%$ week 24, 7\% week 48); APRI, FIB-4, liver stiffness further improved significantly between week 24 and 48 of follow-up. Among the 77 responders (27 F3, 50 F4) who underwent elastography at baseline and at the end of follow-up, 39 (50.6\%; $18 \mathrm{~F} 3,21 \mathrm{~F} 4)$ exhibited a regression in fibrosis stage. Conclusion. At about one-year from the completion of successful sofosbuvir-based therapy, patients with post-LT HCV and severe fibrosis experienced a long-term liver function improvement accompanied by a regression of fibrosis stage in half of them.
\end{abstract}

\title{
Keywords
}

Liver transplantation; HCV recurrence; direct-acting antivirals; non-invasive fibrosis tests; survival. 


\section{Key point box}

- 126 patients with post liver transplant HCV and severe fibrosis (30 F3 and 96 F4 Metavir) received a sofosbuvir-based antiviral therapy. The median follow-up after therapy was 461 days.

- All the $30 \mathrm{~F} 3$ patients and 72 of the 96 cirrhotics (75\%) achieved a sustained virological response at week 24 after treatment.

- HCV-eradicated patients showed a distinct amelioration in AST-to-platelet-ratioindex, FIB-4 and liver stiffness at week 24 after therapy, which persisted in F3 patients and further improved in F4 ones at week 48.

- In transient elastography, the fibrosis stage regressed in half of the HCV-eradicated patients at week 48 after therapy. 


\section{INTRODUCTION}

Hepatitis $\mathrm{C}$ virus (HCV) is the leading cause of cirrhosis and hepatocellular carcinoma and the most common indication for liver transplantation (LT) in Europe and United States [1]. Infection recurs almost universally in patients who have detectable serum HCV RNA at LT and it is the main cause of graft failure and patient death [2]. Successful HCV eradication is associated with reduced disease progression, and improved liver allograft and recipient survival [3].

Until few years ago, pegylated-interferon (Peg-IFN) with ribavirin was the only treatment for HCV recurrence post-LT; however its use was limited by poor efficacy and beset with significant adverse events [4]. The addition of first generation protease inhibitors, boceprevir and telaprevir, doubled the rates of sustained virological response (SVR) at 12 weeks (SVR12) in HCV genotype 1, but was aggravated by unacceptable toxicity and drug-drug interactions [5]. A major breakthrough has been the advent of second generation direct-acting antiviral agents (DAAs), which interact with different HCV functions and have markedly improved antiviral efficacy and tolerability [6], leading to HCV eradication also in patients with severe recurrent hepatitis C after LT.

The therapeutic potential of the prototype sofosbuvir was quickly perceived; the combinations of sofosbuvir with ribavirin \pm Peg-IFN and of sofosbuvir with simeprevir \pm ribavirin were reported in early international studies to achieve $59 \%$ to $88 \%$ SVR12 rates $[7,8]$. A sofosbuvir compassionate use program was activated in Italy between June 2014 and December 2014; simeprevir became available later, in February 2015.

The primary target of early studies was the virologic efficacy and the tolerability of DAAs in order to meet the urgent demand from transplant patients. In this single-center study, we report the clinical outcome of therapy in a large series of patients with severe fibrosis due to hepatitis $\mathrm{C}$ recurrence; the majority were treated with sofosbuvir plus ribavirin, a minority with sofosbuvir plus simeprevir plus ribavirin. We focused on the changes in liver function 
parameters and in non-invasive tests of fibrosis up to 48 weeks after the end of successful treatment.

\section{PATIENTS AND METHODS}

\section{Study population}

Out of 273 surviving HCV recipients with persistent viremia following LT performed in Turin (Italy) since January 2000, between June 2014 and May 2015 we enrolled 126 patients with recurrent hepatitis $C$ who exhibited a Metavir staging score $\geq 3$, and a creatinine clearance $\geq 30 \mathrm{~mL} / \mathrm{min}$. The severity of fibrosis was determined within 6 months from treatment by liver biopsy or by transient elastography [liver stiffness (LS) cut-off values of 9.5 kilopascal ( $\mathrm{kPa}$ ) and $12.5 \mathrm{kPa}$ corresponding, respectively, to Metavir F3 and F4 fibrosis stages] [9]. Only transient elastography measurements with a success rate of at least $60 \%$ were considered valid.

From June 2014 to December 2014 patients were treated in the context of the sofosbuvir national compassionate use program; each treatment was approved by the local ethic committee (Comitato Etico Interaziendale A.O.U. Città della Salute e della Scienza di Torino-A.O. Ordine Mauriziano-A.S.L. TO1) and the patients provided written informed consent. By the Italian legislation, Regional Transplantation Centers are the lawful custodians of the recipient/donor biomedical data for clinical and for research purposes. All study procedures were compliant with the ethical standards of the 2000 Declaration of Helsinki as well as the Declaration of Istanbul 2008.

The demographic and baseline characteristics of the study population and their donors are shown in Table 1. 


\section{Study protocol}

The treatment regimens consisted of sofosbuvir $400 \mathrm{mg}$ once daily and ribavirin twice daily (at a dose of $1000 \mathrm{mg}$ daily with body weight $<75 \mathrm{~kg}$, or $1200 \mathrm{mg}$ daily with body weight $\geq 75 \mathrm{~kg}$, adjusted according to haemoglobin level and renal function) for 24 weeks, or sofosbuvir $400 \mathrm{mg}$ once daily, ribavirin twice daily (weight-based) and simeprevir $150 \mathrm{mg}$ once daily for 12 weeks. Epoetin-alfa at 20,000-40,000 units/week was administered subcutaneously to patients with haemoglobin levels $<10 \mathrm{~g} / \mathrm{dL}$.

Patients underwent clinical and laboratory assessment [HCV RNA by polymerase chain reaction technique with AmpliPrep ${ }^{\circledR} / \mathrm{COBAS}$ TaqMan ${ }^{\circledR} \mathrm{HCV}$ version 2 (Roche Molecular Systems, Inc., Branchburg, NJ, US), complete blood cell count, liver and kidney function tests, serum electrolytes, serum levels of immunosuppressive drugs] at baseline, weekly for the first month, then monthly while on treatment and at 1, 3, 6 and 12 months after the end of therapy (EOT). No viral resistance monitoring was performed.

At baseline, all patients were tested for HCV genotype (reverse hybridization line probe assay, INNO-LIPA, Innogenetics, Ghent, Belgium), Interleukin 28B (IL28B) rs12979860 C/T and rs8099917 T/G polymorphisms (Custom TaqMan ${ }^{\circledR}$ Allelic Discrimination Kit, Life Technologies Applied Biosystems $^{\circledR}$, Thermo Fisher Scientific, Waltham, MA, US) and serum $25-\mathrm{OH}$ vitamin D level (fully automated chemiluminescence immunoassay, ADVIA Centaur $^{\circledR}$ Vitamin D Total assay, Siemens Healthcare Diagnostics Inc., Tarrytown, NY, USA). Human leukocyte antigen (HLA) DRB1 typing was collected as well (performed before transplant, by serology methods until 2006 and then by molecular low-resolution), paying particular attention to HLA-DRB1*11 phenotype [10]. Adverse events (AE), drug dose modifications, use of growth factors, blood transfusions, and need for hospitalisation during treatment were recorded.

To assess the changes in fibrosis evolution in virological responders, transient elastography was performed at 24 and 48 weeks from EOT. The FIB-4 index and the 
aspartate aminotransferase (AST) to platelet ratio index (APRI) were calculated at baseline and 24 and 48 weeks after EOT on fasting blood samples. The following formulas were used. FIB-4=[age (years) $\times$ AST (IU/L)]/[platelets $\left(10^{9} / \mathrm{L}\right) \times$ alanine aminotransferase $\left.(\mathrm{ALT})^{1 / 2}(\mathrm{IU} / \mathrm{L})\right]$; a score of $<1.45$ and $>3.25$ should identify patients who, respectively, have moderate or significant fibrosis [11]. APRI=[AST (IU/L)/upper limit of normal (IU/L)] $x$ $100 /$ platelets $\left(10^{9} / \mathrm{L}\right)$; severe fibrosis was considered unlikely with an APRI $\leq 0.7$ and cirrhosis likely with an APRI $\geq 1$ [12]. Upper limits of normal for our laboratory are: ALT 40 IU/L for male and $35 \mathrm{IU} / \mathrm{L}$ for female; AST $45 \mathrm{IU} / \mathrm{L}$ for male and $30 \mathrm{IU} / \mathrm{L}$ for female. Survival data were collected on May 31, 2016.

\section{Study endpoints}

Primary endpoints of the study were survival and clinical outcome of patients who had achieved a sustained virological response at week 24 after EOT (SVR24). To this purpose we considered the variations from baseline to weeks 24 and 48 of: i) liver function parameters; ii) non-invasive tests of fibrosis (APRI, FIB-4, transient elastography). Secondary endpoint was the safety of antiviral therapy.

\section{Statistical analysis}

Categorical variables were represented as n (\%) and compared using Fisher's exact test. Quantitative variables were shown as median $\left(25^{\text {th }}-75^{\text {th }}\right.$ percentiles $)$ and were compared, as appropriate, by t-test (parametric data), by Mann-Whitney $U$ test (non parametric, unpaired data) or by Wilcoxon signed-rank test (non parametric, paired data). Receiver operating characteristic (ROC) curve analyses were performed to obtain the best possible Model for End-stage Liver Disease (MELD) and Child scores, in order to identify beforehand those patients who achieved SVR24. Data elaboration was performed using 
Prism $^{\circledR}$ version 6 (GraphPad Software Inc., La Jolla, CA, US)and IBM SPSS version 21 (New York, NY, US).

\section{RESULTS}

Of the 126 patients who entered the study, 118 received sofosbuvir and ribavirin for 24 weeks and 8 were treated with sofosbuvir, simeprevir and ribavirin for 12 weeks. In the former group, 116 completed the full course of therapy; 2 patients died while on treatment (on day 93 and 151), 4 following the EOT (on day 41, 143, 166 and 328). The median follow-up of the surviving patients after EOT was 461 days (range 278-515 days).

All patients except 1 were Caucasian. Forty-seven percent of the grafts came from donors $\geq 65$ year old and/or with macrovesicular steatosis $\geq 15 \%$ (suboptimal graft) [13], $8 \%$ from hepatitis $\mathrm{B}$ core antibody positive and $7.1 \%$ from $\mathrm{HCV}$ antibody positive donors.

Two patients had been treated with a first-generation HCV protease-inhibitor before LT. The baseline stage of hepatic fibrosis $(F)$ was evaluated in 28 patients by liver biopsy according to Metavir score (3 F3, 25 F4), in 12 patients by combined liver biopsy and transient elastography (12 F4) and in 86 patients by transient elastography only (27 F3 and 59 F4). The median LS was: $11 \mathrm{kPa}$ [interquartile 10.1-12] in the F3 group and 21.8 kPa [17-34.8] in the F4 group. Thirty-four patients had diabetes mellitus, 33 were insulin dependent at the beginning of antiviral therapy.

The 8 patients treated with sofosbuvir, simeprevir and ribavirin were immunosuppressed with tacrolimus. The HCV genotype was $1 \mathrm{a}$ in two and $1 \mathrm{~b}$ in six. Seven patients had fibrosis Metavir F3 and 1 F4 (Child A5, MELD 7), with median HCV RNA $6.56 \log _{10} \mathrm{IU} / \mathrm{mL}$. Further demographic and baseline characteristics are presented in Table 1.

Four weeks after antiviral therapy was started, a statistically significant decrease was observed in the AST and ALT median values from $67 \mathrm{IU} / \mathrm{L}$ [45-114] to $24 \mathrm{IU} / \mathrm{L}$ [18-33], p $<0.001$, and $58 \mathrm{IU} / \mathrm{L}$ [40-100] to $18 \mathrm{IU} / \mathrm{L}$ [13-26], $\mathrm{p}<0.001$, respectively. Transaminase 
values normalized (Figure S1) and remained normal throughout the follow-up in all eradicated patients but nine, who had persistently elevated enzymes (less than twice upper limit of normal). Six of these latter patients underwent a liver biopsy which showed minimal histological changes without evidence of rejection.

The overall SVR24 rate was $81.0 \%$ (102/126 patients, 95\% confidence interval $73.2-86.9)$ by intention to treat (ITT) analysis. The SVR24 rate was significantly higher in F3 compared with F4 patients $(30 / 30,100 \%$ vs $72 / 96,75 \%, p=0.001)$; in patients tolerating $\geq 80 \%$ of the full weight-based ribavirin dose $(n=104)$ compared with those tolerating $<80 \%$ of the dose $(n=22)(85.6 \%$ vs $59.0 \%, p=0.01)$ and in patients with preserved liver function (MELD $\leq 11$ and Child $\leq$ A6, according to ROC curves) (Figure S2).

Two cirrhotic patients who became HCV RNA negative on therapy, died for multiresistant bacteria-related septic shock; two others died of liver failure after the EOT: one, 68 years old, had achieved a sustained virological response at week 4 after EOT, the other, 44 years old, had achieved SVR12 and was on the list for retransplantation.

Twenty patients experienced a relapse of $\mathrm{HCV}(17$ at week 4, 2 at week 12 and 1 at week 24 of follow-up; the latter cleared HCV RNA at week 4 on therapy and normalized transaminases at week 1). All relapsers were cirrhotic (9 patients Child A, 10 Child B and 1 Child C). They had a median pre-treatment MELD of 13 and had received sofosbuvir and ribavirin; the majority of them were IL28B non-C/C genotype (80\%) and experienced to previous antiviral therapy (65\%) (Tables S1 and S2). Basal median LS was $28.0 \mathrm{kPa}$ in the relapsers and $20.4 \mathrm{kPa}$ in the cirrhotics who achieved viral eradication $(p=0.01)$. APRI and FIB-4 were significantly higher in relapsers compared with responders at baseline (3.27 vs 1.92 and 8.45 vs 5.95, respectively; $p<0.01$ for both) (Table 2 ).

From baseline to EOT, the median Child improved in relapsers from B7 to A6 $(p=0.014)$, while the median MELD remained 13. Two patients died at 138 and 160 days after relapse due to progressive end-stage liver disease. Seventeen patients started a second treatment 
course, at a median of 17 weeks (range 5-43 weeks) from the relapse. No significant variation in liver function tests was observed between relapse and retreatment. One patient refused retreatment.

Overall, in the cirrhotic group, 20 patients (20.8\%) had a stable MELD from baseline to the last available follow-up visit and $43(44.8 \%)$ improved the score of at least 1 point; 46 patients (47.9\%) had a stable Child and 35 (36.5\%) improved the score of at least 1 point.

\section{Clinical outcomes in patients who achieved viral eradication}

In the F3 patients, liver and kidney function remained normal (Table S3). APRI and FIB-4 were significantly lower at week 24 after EOT, compared with pre-treatment median values ( 1.07 to $0.41, p<0.001$, and 3.26 to $2.08, p<0.001$, respectively); these indexes remained stable in the 28 patients who reached 48 weeks of follow-up (Figure 1, Panels A and C). LS (available in 27 patients) improved from $11.0 \mathrm{kPa}$ [10.1-12.0] to $7.7 \mathrm{kPa}$ [6.6-10.2], p $<0.001$, at week 24 and remained stable at week 48 (7.8 kPa [7.0-10.6], $p=0.65$ vs week

24) (Figure 2, Panel A). In 18 patients (66.7\%) LS decreased below the $9.5 \mathrm{kPa}$ cut-off for F3 at week 48.

At week 24 after EOT, the 72 F4 patients who had eradicated HCV, exhibited a significant improvement in platelets count, bilirubin and albumin levels, degree of ascites and Child score at pair wise comparison with baseline values [the rate of Child A was $77.8 \%(56 / 72)$ at baseline and $88.9 \%(64 / 72)$ at week 24 of follow-up]; kidney function and MELD score did not vary. Seventy-one of the 72 responders reached 48 weeks of follow-up and showed a further significant increase in platelets count; albumin, ascites, and Child score [93\% (66/71) Child A] remained stable (Table 3).

Both APRI and FIB-4 decreased significantly at week 24 after EOT compared with baseline values (1.92 to $0.55, p<0.001$, and 5.95 to $3.15, p<0.001$, respectively), and further 
decreased at week 48, compared with 24 week values (APRI 0.51, p <0.001; FIB-4 3.12, p $<0.001$ ) (Figure 1, Panels B and D). Fifty-one of the 72 patients underwent transient elastography at baseline and at SVR24; the median LS improved from $20.4 \mathrm{kPa}$ [16.033.8] to $17.5 \mathrm{kPa}$ [11.1-25.7], $\mathrm{p}<0.001$ (Figure 2, Panel B). Fifty patients underwent transient elastography also at week 48 after EOT; the median LS (14.0 kPa [10.0-21.3]) significantly decreased compared with week $24(p=0.001)$; in 21 of them $(42 \%)$ the LS fell below the $12.5 \mathrm{kPa}$ cut-off.

In $39(50.6 \%)$ of the 77 F3-F4 responders pooled together, the fibrosis stage had regressed in transient elastography by at least one Metavir stage at week 48 after EOT. No patients developed hepatocellular carcinoma in the period of observation.

Thirteen out of the 20 cirrhotic responders with large oesophageal varices at baseline, underwent a second 48 week follow-up endoscopy; no change in the size of varices was observed.

\section{Safety}

$\mathrm{AE}$ are summarized in Table $\mathbf{S 4 .}$

During therapy, seven cirrhotic patients were hospitalised: two patients for grade 3 encephalopathy, two for severe variceal bleeding and three for sepsis in decompensated liver disease. Six cirrhotic patients died of complications of end-stage liver disease, unrelated to antiviral therapy.

Three patients discontinued ribavirin for less than 1 week due to diffuse macular skin rash and no one discontinued therapy permanently due to $A E$.

Because of anemia and/or compromised renal function, $34.1 \%(43 / 126)$ of the patients were treated with a non weight-based escalating dose of ribavirin, starting from $400 \mathrm{mg}$ daily and $11.1 \%(14 / 126)$ required dosage reduction; however, $82.5 \%(104 / 126)$ received at least $80 \%$ of the weight-based dosage. Median daily ribavirin dose was $1000 \mathrm{mg}$ from 
baseline to EOT and median haemoglobin level declined from $12.8 \mathrm{~g} / \mathrm{dL}$ at baseline to 10.9 $\mathrm{g} / \mathrm{dL}$ at week 4 and remained stable around $10.8 \mathrm{~g} / \mathrm{dL}$ from week 8 to week 24 (Figure S3). In 60 patients (47.6\%) we measured at least one haemoglobin level of less than 10 $\mathrm{g} / \mathrm{dL}$ and in $12(9.5 \%)$ of less than $8.5 \mathrm{~g} / \mathrm{dL} ; 45$ patients $(35.7 \%)$ received epoetin and 14 $(11.1 \%)$ required blood transfusion.

Minimal dose adjustments in the calcineurin inhibitors were required throughout the treatment in $64 \%$ of the patients $(27 \%$ increased, $12 \%$ decreased and $25 \%$ both decreased and increased the dose) to maintain stable trough levels. No biopsy-proven acute rejection occurred.

\section{DISCUSSION}

The advent of highly effective and well tolerated DAAs against the HCV is revolutionizing the outcome of LT for hepatitis C. In this setting, early treatment after surgery is expected to be the most cost-effective strategy for patients who are still viremic at LT $[10,14]$, nevertheless DAAs are providing rescue also to residual cohorts of re-infected transplant recipients who developed advanced $\mathrm{HCV}$ disease $[7,8,15]$. When sofosbuvir became available in Italy in June 2014 , these patients received priority access to the new therapy through a compassionate use program. A majority were treated with sofosbuvir and ribavirin; a small minority received the combination of sofosbuvir, ribavirin and simeprevir, which became available almost at the end of the enrollment period.

After a median follow-up of 461 days from EOT, $102(81 \%)$ of our 126 patients are free of HCV and clinically stable. SVR was obtained also in a high proportion (95\%) of the 21 patients with genotype 3 , though this genotype is the most difficult to treat in the ordinary clinical context. All treatment failures, excluding deceased patients, were due to virologic relapses and all relapses, except one, occurred within the first 12 weeks after EOT. Response to therapy was influenced by the stage of fibrosis and by intolerance to ribavirin: 
$75 \%$ of the patients with cirrhosis achieved an SVR compared to $100 \%$ of those with F3 fibrosis and among the 22 patients who tolerated less than $80 \%$ of the optimal ribavirin dosage, only $59 \%$ obtained an SVR. As expected, anemia was the main complication of ribavirin.

Considering the higher proportion of patients with cirrhosis in this study, our results appear better than the $70 \%$ rate of SVR12 reported by Charlton [16] in a smaller series of 40 patients recruited in a multicenter study, of whom only $40 \%$ were cirrhotics; possibly the management of the patients in a single center adopting a common approach may lead to more uniform results. Though our results are distinctly better than the $43 \%$ response reported by Forns et al [7], their series included a mixed population of compensated and decompensated cirrhotics in which a substantial number of patients had a higher MELD score and more severe disease than our patients.

There were in our study six deaths due to complications of the liver disease, two occurring in patients who had cleared the HCV. The deceased patients had a higher MELD score, ranging from 14 to 19 , in confirmation that a deteriorated liver function may prevent clinical improvement despite the antiviral efficacy of treatment [17]; vice versa a preserved liver function expressed by a Child Pugh score $\leq 6$ and a MELD $\leq 11$ was associated with SVR. In our 72 cirrhotic responders, SVR24 was associated with the normalization of aminotransferases in almost all patients, accompanied by a decrease of bilirubin and an increase of albumin levels; renal function deteriorated in none and ascites disappeared in three quarters of cases. The platelet count increased throughout therapy, presumably related to the administration of epoetin and to an increased production of endogenous erythropoietin, secondary to ribavirin-induced anemia [18]. The platelet number diminished to baseline values at week 12 post-therapy, but then climbed back significantly at week 24 and further increased at week 48; this trend and the regression of ascites might be expression of an initial reduction in portal hypertension. 
The APRI and FIB-4 indexes significantly improved throughout the follow-up; as aminotransferases and platelets are major components for their calculation, the improvement can be explained by the normalization of the enzymes and by the increase in the platelet count.

LS values were significantly reduced at the $6^{\text {th }}$ month of follow-up; this early improvement presumably resulted for a large extent from the decrease of inflammation and edema which may artificially alter elastography. Interestingly, however, LS values showed a further significant decrease during the subsequent 6 month follow-up in patients with cirrhosis. Considering that artefacts due to liver inflammation could no longer be implicated, it is plausible that the observed continuing decrease of LS after the SVR24 corresponded to a genuine progressive reduction of fibrosis. In agreement with previous findings of fibrosis reversion in non-transplant patients with HCV cirrhosis who responded to interferontherapy [19-21], our data would suggest that regression of fibrosis starts early and may become already measureable by transient elastography in the first year after therapy. In conclusion, our experience in a large single center LT cohort of advanced recurrent HCV disease, confirms the major therapeutic impact of sofosbuvir with ribavirin, extending also to difficult-to-treat genotype 3 patients.

We acknowledge that a new wave of more efficacious DAA combinations is now available to treat hepatitis $C[8,15,22]$. Nevertheless, this study provides a paradigm of the excellent clinical outcome and of the potential for disease reversibility after successful DAA therapy in the transplant setting.

\section{ACKNOWLEDGEMENTS}

No funding was obtained for this study. 


\section{REFERENCES}

[1] Adam R, Karam V, Delvart V. Evolution of indications and results of liver transplantation in Europe. A report from the European Liver Transplant Registry (ELTR). J Hepatol 2012;57:675-688.

[2] Forman LM, Lewis JD, Berlin JA, Feldman HI, Lucey MR. The association between hepatitis $\mathrm{C}$ infection and survival after orthotopic liver transplantation. Gastroenterology 2002;122:889-896.

[3] Crespo G, Marino Z, Navasa M, Forns X. Viral hepatitis in liver transplantation. Gastroenterology 2012;142:1373-1383.

[4] Berenguer M. Systematic review of the treatment of established recurrent hepatitis C with pegylated interferon in combination with ribavirin. J Hepatol 2008;49:274-287.

[5] Coilly A, Roche B, Dumortier J, Leroy V, Botta-Fridlund D, Radenne S, et al. Safety and efficacy of protease inhibitors to treat hepatitis $\mathrm{C}$ after liver transplantation: a multicenter experience. J Hepatol 2014;60:78-86.

[6] EASL recommendations on treatment of hepatitis C 2015. J Hepatol 2015;63:199236.

[7] Forns X, Charlton M, Denning J, McHutchison JG, Symonds WT, Brainard D, et al. Sofosbuvir compassionate use program for patients with severe recurrent hepatitis $\mathrm{C}$ after liver transplantation. Hepatology 2015;61:1485-1494.

[8] Brown RS Jr, O'Leary JG, Reddy KR, Kuo A, Morelli GJ, Burton JR Jr, et al. Interferon-free therapy for genotype 1 hepatitis $C$ in liver transplant recipients: real world experience from HCV-TARGET. Liver Transpl 2016;22:24-33.

[9] Castera L, Forns X, Alberti A. Non-invasive evaluation of liver fibrosis using transient elastography. J Hepatol 2008;48:835-847.

[10] Romagnoli R, Martini S, Tandoi F, Dell Olio D, Magistroni P, Bertinetto FE, et al. Early reduced liver graft survival in hepatitis $\mathrm{C}$ recipients identified by two combined 
genetic markers. Transpl Int 2016; doi: 10.1111/tri.12795.

[11] Vallet-Pichard A, Mallet V, Nalpas B, Verkarre V, Nalpas A, Dhalluin-Venier V, et al. FIB-4: an inexpensive and accurate marker of fibrosis in HCV infection. Comparison with liver biopsy and fibrotest. Hepatology 2007;46:32-36.

[12] Wai CT, Greenson JK, Fontana RJ, Kalbfleisch JD, Marrero JA, Conjeevaram HS, et al. A simple non-invasive index can predict both significant fibrosis and cirrhosis in patients with chronic hepatitis C. Hepatology 2003;38:518-526.

[13] Salizzoni M, Franchello A, Smedile A, David E, Barbui A, Torrani M, et al. Marginal grafts: finding the correct treatment for fatty livers. Transpl Int 2003;16:486-493.

[14] Russo FP, Zanetto A, Burra P. Timing for treatment of HCV recurrence after liver transplantation: the earlier the better. Transpl Int 2016;29:694-697.

[15] Charlton M, Everson GT, Flamm SL, Kumar P, Landis C, Brown RS, et al. Ledipasvir and sofosbuvir plus ribavirin for treatment of HCV infection in patients with advanced liver disease. Gastroenterology 2015;149:649-659.

[16] Charlton M, Gane E, Manns MP, Brown RS Jr, Curry MP, Kwo PY, et al. Sofosbuvir and ribavirin for treatment of compensated recurrent hepatitis $C$ virus infection after liver transplantation. Gastroenterology 2015;148:108-117.

[17] Pellicelli AM, Montalbano M, Lionetti R, Durand C, Ferenci P, D’Offizi G, et al. Sofosbuvir plus daclatasvir for post-transplant recurrent hepatitis C: potent antiviral activity but no clinical benefit if treatment is given late. Dig Liver Dis 2014;46:923-927.

[18] Kobayashi T, Hige S, Terashita K, Nakai M, Horimoto H, Sho T, et al. Anemia and thrombocytosis induced by ribavirin monotherapy in patients with chronic hepatitis $\mathrm{C}$. J Gastroenterol 2012;47:1228-1237.

[19] Poynard T, McHutchison J, Manns M, Trepo C, Lindsay K, Goodman Z, et al. Impact of pegylated interferon alfa-2b and ribavirin on liver fibrosis in patients with chronic hepatitis C. Gastroenterology 2002;122:1303-1313. 
Page 19 of 49

Liver International

1
2
3
4
5
6
7
8
9
10
11
12
13
14
15
16
17
18
19
20
21
22
23
24
25
26
27
28
29
30
31
32
33
34
35
36
37
38
39
40
41
42
43
44
45
46
47
48
49
50
51
52
53
54
55
56
57
58
59
60

[20] EASL-ALEH clinical practice guidelines: non-invasive tests for evaluation of liver disease severity and prognosis. European Association for the Study of the Liver. J Hepatol 2015;63:237-264.

[21] D'Ambrosio R, Aghemo A, Fraquelli M, Rumi MG, Donate MF, Paradis V, et al. The diagnostic accuracy of Fibroscan ${ }^{\circledR}$ for cirrhosis is influenced by liver morphometry in HCV patients with a sustained virological response. J Hepatol 2013;59:251-256.

[22] Foster GR, Irving WL, Cheung MC, Walker AJ, Hudson BE, Verna S, et al. HCV Research UK. Cohort study of the impacts of direct acting antiviral therapy in patients with chronic hepatitis C and decompensated cirrhosis. J Hepatol 2016;64:1224-1231. 
Table 1. Demographic and baseline characteristics of recipients and donors

\begin{tabular}{|c|c|}
\hline \multicolumn{2}{|l|}{ Recipient features $(N=126)$} \\
\hline Age (years) & $60[54-66]$ \\
\hline Male & $99(78.6 \%)$ \\
\hline Body Mass Index $\left(\mathrm{Kg} / \mathrm{m}^{2}\right)$ & $24.6[22.5-26.9]$ \\
\hline $25-\mathrm{OH}$ vitamin $\mathrm{D}^{\wedge}(<20 \mathrm{ng} / \mathrm{mL})$ & $71(59.2 \%)$ \\
\hline HLA-DRB1*11 positive phenotype & $34(27.0 \%)$ \\
\hline \multicolumn{2}{|l|}{ IL28B genotype } \\
\hline rs $12979860 \mathrm{C} / \mathrm{C}$ & $32(25.4 \%)$ \\
\hline rs $8099917 \mathrm{~T} / \mathrm{T}$ & $59(46.8 \%)$ \\
\hline Previous antiviral treatment & $83(65.9 \%)$ \\
\hline Antiviral treatment after LT & $57(45.2 \%)$ \\
\hline \multicolumn{2}{|l|}{ Immunosuppressive drug } \\
\hline CsA - Tac - MMF - other & $50(39.7 \%)-56(44.4 \%)-56(44.4 \%)-5(4.0 \%)$ \\
\hline Time since LT (years) & $4.3[2.3-7.2]$ \\
\hline \multicolumn{2}{|l|}{ HCV genotype } \\
\hline $1 a-1 b-1$ (unspecified) & $17(13.5 \%)-70(55.6 \%)-6(4.8 \%)$ \\
\hline $2-3-4$ & $6(4.8 \%)-21(16.7 \%)-6(4.8 \%)$ \\
\hline Baseline HCV RNA $\left(\log _{10} \mathrm{IU} / \mathrm{mL}\right)$ & $6.40[6.10-6.66]$ \\
\hline$\geq 10^{6} \mathrm{IU} / \mathrm{mL}$ & $101(80.2 \%)$ \\
\hline
\end{tabular}

\section{Corresponding Metavir fibrosis stage}
$\mathrm{F} 3-\mathrm{F} 4$
$30(23.8 \%)-96(76.2 \%)$

\begin{tabular}{lc}
\hline Haemoglobin $(\mathrm{g} / \mathrm{dL})$ & $12.8[11.4-14.4]$ \\
\hline Platelets count $\left(10^{9} / \mathrm{L}\right)$ & $99[67-140]$ \\
\hline Liver enzymes $(\mathrm{IU} / \mathrm{L})$ & $60[40-101]$ \\
Aspartate aminotransferase & $69[45-116]$ \\
Alanine aminotransferase & $75[41-152]$ \\
Y-Glutamyltransferase &
\end{tabular}


Numerical variables are expressed as median [Q1-Q3]

Categorical variables are expressed as numbers (prevalence, \%)

${ }^{\wedge} 6$ missing data

${ }^{*}$ Chronic Kidney Disease Epidemiology Collaboration equation

$\S$ In 96 cirrhotic patients

\#According to Salizzoni et al, Transpl Int 2003

Abbreviations: CsA, cyclosporine; D-MELD, donor age x recipient MELD; eGFR, estimated glomerular filtration rate; F, fibrosis; HCV, hepatitis C virus; HLA, human leukocyte antigen; IL28B, Interleukin 28B; LT, liver transplantation; MELD, Model for End-stage Liver Disease; MMF, mycophenolate mofetil; Tac, tacrolimus. 
Table 2. Liver function tests and non-invasive markers of fibrosis at baseline and at the end of therapy in responder versus relapser cirrhotic patients

\begin{tabular}{lccc}
\hline BASELINE & $\begin{array}{c}\text { Responders } \\
(\mathbf{n = 7 2})\end{array}$ & $\begin{array}{c}\text { Relapsers } \\
(\mathbf{n}=\mathbf{2 0})\end{array}$ & $\mathbf{p}^{\#}$ \\
\hline APRI & $1.92[1.00-3.27]$ & $3.27[1.72-5.51]$ & 0.007 \\
FIB-4 & $5.95[3.60-11.10]$ & $8.45[7.17-16.08]$ & 0.009 \\
Liver stiffness ${ }^{*}(\mathbf{k P a})$ & $20.4[16.0-33.8]$ & $28.0[21.6-44.4]$ & 0.01 \\
Child score & $5[5-6]$ & $7[6-8]$ & 0.002 \\
MELD score & $10[8-12]$ & $13[9-16]$ & 0.02 \\
& & & \\
\hline EOT & Responders & $\mathbf{R e l a p s e r s}$ & $\mathbf{p}^{\#}$ \\
\hline APRI & $(\mathbf{n}=\mathbf{7 2})$ & $0.83[0.57-1.22]$ & 0.02 \\
FIB-4 & $0.46[0.29-0.76]$ & $4.80[3.71-7.42]$ & 0.01 \\
Liver stiffness ${ }^{*}(\mathbf{k P a})$ & $3.25[1.90-4.30]$ & $23.4[18.3-39.0]$ & 0.09 \\
Child score & $17.5[11.1-25.7]$ & $6[5-7]$ & 0.19 \\
MELD score & $5[5-6]$ & $13[11-17]$ & 0.05 \\
\hline
\end{tabular}

Numerical variables are expressed as median [Q1-Q3]

*Liver stiffness was available at baseline in 51 responders and 17 relapsers; at EOT in 51 responders and 13 relapsers

\#p-values calculated according to Wilcoxon test for paired data

Abbreviations: APRI, AST to platelet ratio index; EOT, end of therapy; kPa, kilopascal; MELD, Model for Endstage Liver Disease. 
Table 3. Clinical and biochemical variations in long-term responder cirrhotic patients

\begin{tabular}{|c|c|c|c|c|c|c|c|}
\hline & $\begin{array}{l}\text { Baseline } \\
(\mathrm{N}=72)\end{array}$ & $\begin{array}{c}\text { EOT } \\
(N=72)\end{array}$ & $\begin{array}{l}\text { SVR12 } \\
(N=72)\end{array}$ & $\begin{array}{l}\text { SVR24 } \\
(N=72)\end{array}$ & $\begin{array}{l}\text { SVR48 } \\
\left(\mathrm{N}=71^{\circ}\right)\end{array}$ & $p^{\wedge}$ & $p^{\S}$ \\
\hline AST (IU/L) & 69 [47-97] & $23[20-28]$ & 27 [22-35] & $24[18-32]$ & $21[18-28]$ & $<0.001$ & 0.13 \\
\hline ALT (IU/L) & 59 [41-90] & $15[11-23]$ & $18[14-27]$ & $16[12-25]$ & $17[12-27]$ & $<0.001$ & 0.02 \\
\hline Bilirubin (mg/dL) & $1.1[0.8-1.5]$ & $1.4[1.1-2.2]$ & $0.9[0.6-1.2]$ & $0.8[0.6-1.2]$ & $0.9[0.6-1.1]$ & $<0.001$ & 0.74 \\
\hline INR & $\begin{array}{c}1.06 \\
{[0.99-1.20]}\end{array}$ & $\begin{array}{c}1.11 \\
{[1.07-1.24]}\end{array}$ & $\begin{array}{c}1.09 \\
{[1.02-1.24]}\end{array}$ & $\begin{array}{c}1.08 \\
{[1.02-1.22]}\end{array}$ & $\begin{array}{c}1.11 \\
{[1.07-1.23]}\end{array}$ & 0.03 & 0.007 \\
\hline Albumin (g/dL) & $3.9[3.6-4.3]$ & $4.1[3.8-4.4]$ & $4.1[3.7-4.3]$ & $4.2[3.8-4.4]$ & $4.3[4.0-4.5]$ & 0.02 & 0.31 \\
\hline Creatinine (mg/dL) & $1.1[0.9-1.3]$ & $1.1[0.9-1.3]$ & $1.0[0.9-1.3]$ & $1.1[1.0-1.3]$ & $1.1[0.9-1.3]$ & 0.36 & 0.12 \\
\hline eGFR* (mL/min) & 68 [52-84] & $64[52-80]$ & $67[53-83]$ & $63[52-78]$ & $62[52-82]$ & 0.26 & 0.25 \\
\hline Platelets $\left(10^{9} / \mathrm{L}\right)$ & 99 [65-134] & $131[86-185]$ & 99 [64-133] & 108 [72-142] & 112 [68-144] & 0.002 & 0.01 \\
\hline Ascites & $22(30.6 \%)$ & $12(16.7 \%)$ & $8(11.1 \%)$ & $7(9.7 \%)$ & $7(9.9 \%)$ & 0.003 & $>0.99$ \\
\hline Encephalopathy & $5(6.9 \%)$ & $4(5.6 \%)$ & $3(4.2 \%)$ & $3(4.2 \%)$ & $2(2.8 \%)$ & 0.72 & $>0.99$ \\
\hline MELD score & 10 [8-12] & 11 [9-14] & 9 [8-11] & 9 [8-11] & 10 [8-11] & 0.34 & 0.92 \\
\hline Child score & $5[5-6]$ & $5[5-6]$ & $5[5-6]$ & $5[5-5]$ & $5[5-5]$ & $<0.001$ & 0.68 \\
\hline
\end{tabular}

Numerical variables are expressed as median [Q1-Q3]

Categorical variables are expressed as numbers (prevalence,\%)

p-values calculated according to Wilcoxon test for paired data for numerical variables and according to Fisher's exact test for categorical variables

${ }^{\circ} 71$ out of 72 cured patients reached 48 weeks of follow-up after therapy

${ }^{*}$ Chronic Kidney Disease Epidemiology Collaboration equation

^Baseline versus SVR24

§SVR24 versus SVR48

Abbreviations: ALT, alanine aminotransferase; AST, aspartate aminotransferase; eGFR, estimated glomerular filtration rate; EOT, end of therapy; MELD, Model for End-stage Liver Disease; SVR12, sustained virological response at week 12 after end of therapy; SVR24, sustained virological response at week 24 after end of therapy; SVR48, sustained virological response at week 48 after end of therapy. 


\section{FIGURE LEGENDS}

Figure 1. Variations of APRI (Panels A and B) and FIB-4 (Panels C and D) from baseline to weeks 24 and 48 after therapy, in F3 and F4 long-term responders. APRI, AST to platelet ratio index; F, fibrosis according to Metavir; SVR24, sustained virological response at week 24 after therapy; SVR48, sustained virological response at week 48 after therapy.

Figure 2. Variations of liver stiffness from baseline to weeks 24 and 48 after therapy in F3 (Panel A) and F4 (Panel B) long-term responders. F, fibrosis according to Metavir; kPa, kilopascal; SVR24, sustained virological response at week 24 after therapy; SVR48, sustained virological response at week 48 after therapy. 


\title{
Impact of Viral Eradication with Sofosbuvir-based Therapy on the Outcome of Post-Transplant Hepatitis C with Severe Fibrosis
}

\author{
Silvia Martini, ${ }^{1}$ Marco Sacco, ${ }^{1}$ Silvia Strona, ${ }^{1}$ Daniele Arese, ${ }^{1}$ Francesco Tandoi, ${ }^{2}$ \\ Dominic Dell Olio, ${ }^{3}$ Davide Stradella, ${ }^{1}$ Donatella Cocchis, ${ }^{2}$ Stefano Mirabella, ${ }^{2}$ \\ Giorgia Rizza, ${ }^{2}$ Paola Magistroni, ${ }^{3}$ Pamela Moschini, ${ }^{3}$ Antonio Ottobrelli, ${ }^{1}$ \\ Antonio Amoroso, ${ }^{3}$ Mario Rizzetto, ${ }^{1}$ Mauro Salizzoni, ${ }^{2}$ \\ Giorgio M. Saracco, ${ }^{1}$ Renato Romagnoli ${ }^{2}$ \\ ${ }^{1}$ Gastrohepatology Unit \\ ${ }^{2}$ Liver Transplantation Center, General Surgery Unit $2 U$ \\ ${ }^{3}$ Regional Transplantation Center, Piedmont \\ AOU Città della Salute e della Scienza di Torino, University of Turin, Italy
}

\section{Correspondence information}

Silvia Martini, MD

Gastrohepatology Unit, AOU Città della Salute e della Scienza di Torino

Corso Bramante, 88 - 10126 Turin - Italy

Phone: +39 011 6335928; fax: +390116334014

E-mail address: sivima21@hotmail.com

Electronic word count for main body of the manuscript: $4468 \underline{4337}$.

Number of figures: $\underline{2} 4$. Number of tables: $\underline{3} z$. 


\section{List of abbreviations in the order of appearance:}

HCV, hepatitis C virus; LT, liver transplantation; Peg-IFN, pegylated-interferon; SVR, sustained virological response; SVR12, sustained virological response at week 12 after the end of treatment; DAAs, direct-acting antiviral agents; LS, liver stiffness; kPa, kilopascal; EOT, end of therapy; IL28B, Interleukin 28B; HLA, human leukocyte antigen; AE, adverse event; AST, aspartate aminotransferase; APRI, AST to platelet ratio index; ALT, alanine aminotransferase; SVR24, sustained virological response at week 24 after the end of treatment; ROC, receiver operating characteristic; MELD, Model for End-stage Liver Disease; F, fibrosis; ITT, intention to treat. AUC, area under the curve.

\section{Conflict of interest}

MR is an Advisory Board member for AbbVie, BMS, Gilead, Janssen, MSD.

GMS is an Advisory Board member for AbbVie, Gilead and MSD.

$\mathrm{SM}$ is a Regional Advisory Board member for Gilead.

The other Authors who have taken part in this study declared no conflict of interest.

\section{Financial support}

This work was unsupported. 


\section{ABSTRACT}

Background \& Aims. Several studies reporthave shown that the new direct-acting-antivirals (DAAs) induce maintain viral their efficacyeradication also in liver transplant (LT) recipients with severe HCV recurrence. We determined The the clinical impact of Sofosbuvir/Ribavirin in LT therapy through the changes in on liver function and on fibrosis progressionstate at 24 and 48 weeks after treatmentwas evaluated.

Methods. Between 06/2014 and 07/2015, 126 patients (30 F3, 96 F4 Metavir stage) were enrolled to receive sofosbuvir+ribavirin (24 weeks, 118 patients) or sofosbuvir+simeprevir+ribavirin (12 weeks, 8 patients); treatment was initiated at a median time of 4.3 years from LT. Median follow-up after therapy completion was 461 days. Results. All 30 F3 patients achieved a sustained virological response at week 24 after treatment (SVR24), and showed a distinct amelioration of the AST-to-platelet-ratio-index (APRI), FIB-4 and liver stiffness at elastography by week 24 post-therapy, which were maintained at week 48 .

Of the 96 F4 cirrhotic patients, 72 (75\%) achieved SVR24 accompanied by a significant improvement of liver function, which was maintained at week 48 (Child B-C 22\% baseline, $11 \%$ week 24, 7\% week 48); APRI, FIB-4, and liver stiffness further improved significantly between week 24 and 48 of follow-up. Among the 77 responders ( 27 F3, 50 F4) who underwent elastography at baseline and at the end of follow-up, 39 (50.6\%; 18 F3, $21 \mathrm{~F} 4)$ exhibited a regression in fibrosis stage. Conclusion. At about one-year from the completion of successful sofosbuvir-based therapy, patients with post-LT HCV and severe fibrosis experienced a long-term liver function improvement accompanied by a regression of fibrosis stage in half of them. 


\section{Keywords}

Liver transplantation; HCV recurrence; direct-acting antivirals; non-invasive fibrosis tests; survival.

\section{Key point box}

- 126 patients with post liver transplant HCV and severe fibrosis (30 F3 and 96 F4 Metavir) received a sofosbuvir-based antiviral therapy. The Amedian follow-up after therapy was 461 days.

- All the 30 F3 patients and 72/96 72 of the 96 (75\%) cirrhotics $(75 \%)$ are currently alive and achieved a sustained virological response at week 24 after treatment.

- HCV-eradicated cured-patients showed a distinct amelioration in AST-to-plateletratio-index, FIB-4 and liver stiffness at week 24 after therapy, which persisted in F3 patients and further improved in F4 ones at week 48.

- In transient elastography, the fibrosis stage regressed in half of the HCV-eradicated Overall, a fibrosis stage regression evaluated by transient elastography, occurred in half of the patients at week 48 after therapy. 


\section{INTRODUCTION}

Hepatitis $\mathrm{C}$ virus (HCV) is the leading cause of cirrhosis and hepatocellular carcinoma and the most common indication for liver transplantation (LT) in Europe and United States [1]. Infection recurs almost universally in patients who have detectable serum HCV RNA at LT and it is the main cause of graft failure and patient death [2]. Successful HCV eradication is associated with reduced disease progression, and improved liver allograft and recipient survival [3].

Until few years ago, pegylated-interferon (Peg-IFN) with ribavirin was the only treatment for HCV recurrence post-LT; however its use was limited by poor efficacy and beset with significant adverse events [4]. The addition of first generation protease inhibitors, boceprevir and telaprevir, doubled the rates of sustained virological response (SVR) at 12 weeks (SVR12) in HCV genotype 1, but was aggravated by unacceptable toxicity and drug-drug interactions [5]. A major breakthrough has been the advent of second generation direct-acting antiviral agents (DAAs), which interact with different HCV functions and have markedly improved antiviral efficacy and tolerability [6], leading to HCV eradication also in patients with severe recurrent hepatitis C after LT.

The therapeutic potential of the prototype sofosbuvir was quickly perceived; the combinations of sofosbuvir with ribavirin \pm Peg-IFN and of sofosbuvir with simeprevir \pm ribavirin were reported in early international studies to achieve $59 \%$ to $88 \%$ SVR12 rates [7, 8]. A sofosbuvir compassionate use program was activated in Italy between June 2014 and December 2014; simeprevir became available later, in February 2015.

The primary target of early studies was the virologic efficacy and the tolerability of DAAs in order to meet the urgent demand from transplant patients. In this single-center study, we report the clinical outcome of therapy in a large series of patients with severe fibrosis due to hepatitis $\mathrm{C}$ recurrence; the majority were treated with sofosbuvir plus ribavirin, a minority with sofosbuvir plus simeprevir plus ribavirin. We focused on the changes in liver function 
parameters and in non-invasive tests of fibrosis up to 48 weeks after the end of successful treatment. These studies had short follow-ups and did not deal with the long-term clinicaloutcome of LT patients who had responded to therapy. In this single-center study, we collected a large series of patients with post-transplant severe fibrosis due to hepatitis $C$ recurrence; the majority were treated with sofosbuvir plus ribavirin, a minority with sofosbuvir plus simeprevir plus ribavirin. We report the virologic results and focus on the changes observed in liver function parameters and in noninvasive tests of fibrosis up to 48 weeks after the end of successful therapy.

\section{PATIENTS AND METHODS}

\section{Study population}

Out of 273 surviving HCV recipients with persistent viremia following LT performed in Turin (Italy) since January 2000, between June 2014 and May 2015 we enrolled 126 patients with recurrent hepatitis $C$ who exhibited a Metavir staging score $\geq 3$, and a creatinine clearance $\geq 30 \mathrm{~mL} / \mathrm{min}$. The severity of fibrosis was determined within 6 months from treatment by liver biopsy or by transient elastography [liver stiffness (LS) cut-off values of 9.5 kilopascal $(\mathrm{kPa})$ and $12.5 \mathrm{kPa}$ corresponding, respectively, to Metavir F3 and F4 fibrosis stages] [9]. Only transient elastography measurements with a success rate of at least $60 \%$ were considered valid.

From June 2014 to December 2014 patients were treated in the context of the sofosbuvir national compassionate use program; each treatment was approved by the local ethic committee (Comitato Etico Interaziendale A.O.U. Città della Salute e della Scienza di Torino-A.O. Ordine Mauriziano-A.S.L. TO1) and the patients provided written informed consent. By the Italian legislation, Regional Transplantation Centers are the lawful custodians of the recipient/donor biomedical data for clinical and for research purposes. All study procedures were compliant with the ethical standards of the 2000 Declaration of 
Helsinki as well as the Declaration of Istanbul 2008.

The demographic and baseline characteristics of the study population and their donors are shown in Table 1.

\section{Study protocol}

The treatment regimens consisted of sofosbuvir $400 \mathrm{mg}$ once daily and ribavirin twice daily (at a dose of $1000 \mathrm{mg}$ daily with body weight $<75 \mathrm{~kg}$, or $1200 \mathrm{mg}$ daily with body weight $\geq 75 \mathrm{~kg}$, adjusted according to haemoglobin level and renal function) for 24 weeks, or sofosbuvir $400 \mathrm{mg}$ once daily, ribavirin twice daily (weight-based) and simeprevir $150 \mathrm{mg}$ once daily for 12 weeks. Epoetin-alfa at 20,000-40,000 units/week was administered subcutaneously to patients with haemoglobin levels $<10 \mathrm{~g} / \mathrm{dL}$.

Patients underwent clinical and laboratory assessment [HCV RNA by polymerase chain reaction technique with AmpliPrep ${ }^{\circledR}$ /COBAS TaqMan ${ }^{\circledR}$ HCV version 2 (Roche Molecular Systems, Inc., Branchburg, NJ, US), complete blood cell count, liver and kidney function tests, serum electrolytes, serum levels of immunosuppressive drugs] at baseline, weekly for the first month, then monthly while on treatment and at 1, 3, 6 and 12 months after the end of therapy (EOT). No viral resistance monitoring was performed.

At baseline, all patients were tested for HCV genotype (reverse hybridization line probe assay, INNO-LIPA, Innogenetics, Ghent, Belgium), Interleukin 28B (IL28B) rs12979860 C/T and rs8099917 T/G polymorphisms (Custom TaqMan ${ }^{\circledR}$ Allelic Discrimination Kit, Life Technologies Applied Biosystems ${ }^{\circledR}$, Thermo Fisher Scientific, Waltham, MA, US) and serum $25-\mathrm{OH}$ vitamin D level (fully automated chemiluminescence immunoassay, ADVIA Centaur $^{\circledR}$ Vitamin D Total assay, Siemens Healthcare Diagnostics Inc., Tarrytown, NY, USA). Human leukocyte antigen (HLA) DRB1 typing was collected as well (performed before transplant, by serology methods until 2006 and then by molecular low-resolution), paying particular attention to HLA-DRB1*11 phenotype [10]. Adverse events (AE), drug 
dose modifications, use of growth factors, blood transfusions, and need for hospitalisation during treatment were recorded.

To assess the changes in fibrosis evolution in virological responders, transient elastography was performed at 24 and 48 weeks from EOT. The FIB-4 index and the aspartate aminotransferase (AST) to platelet ratio index (APRI) were calculated at baseline and 24 and 48 weeks after EOT on fasting blood samples. The following formulas were used. FIB-4=[age (years) $\times$ AST (IU/L)]/[platelets $\left(10^{9} / \mathrm{L}\right) \times$ alanine aminotransferase $\left.(\mathrm{ALT})^{1 / 2}(\mathrm{IU} / \mathrm{L})\right]$; a score of $<1.45$ and $>3.25$ should identify patients who, respectively, have moderate or significant fibrosis [11]. APRI=[AST (IU/L)/upper limit of normal (IU/L)] $x$ $100 /$ platelets $\left(10^{9} / \mathrm{L}\right)$; severe fibrosis was considered unlikely with an APRI $\leq 0.7$ and cirrhosis likely with an APRI $\geq 1$ [12]. Upper limits of normal for our laboratory are: ALT 40 IU/L for male and $35 \mathrm{IU} / \mathrm{L}$ for female; AST $45 \mathrm{IU} / \mathrm{L}$ for male and $30 \mathrm{IU} / \mathrm{L}$ for female. Survival data were collected on May 31, 2016.

\section{Study endpoints}

Primary endpoints of the study were survival and clinical outcome of patients who had achieved a sustained virological response at week 24 after EOT (SVR24). To this purpose we considered the variations from baseline to weeks 24 and 48 of: i) liver function parameters; ii) non-invasive tests of fibrosis (APRI, FIB-4, transient elastography). Secondary endpoint was the safety of antiviral therapy.

\section{Statistical analysis}

Categorical variables were represented as n (\%) and compared using Fisher's exact test. Quantitative variables were shown as median $\left(25^{\text {th }}-75^{\text {th }}\right.$ percentiles $)$ and were compared, as appropriate, by t-test (parametric data), by Mann-Whitney $U$ test (non parametric, unpaired data) or by Wilcoxon signed-rank test (non parametric, paired data). Receiver 
operating characteristic (ROC) curve analyses were performed to obtain the best possible Model for End-stage Liver Disease (MELD) and Child scores., and non-invasive fibrosistests thresholds, in order to identify beforehand those patients who achieved SVR24. Data elaboration was performed using Prism ${ }^{\circledR}$ version 6 (GraphPad Software Inc., La Jolla, CA, US)and IBM SPSS version 21 (New York, NY, US).

\section{RESULTS}

Of the 126 patients who entered the study, 118 received sofosbuvir and ribavirin for 24 weeks and 8 were treated with sofosbuvir, simeprevir and ribavirin for 12 weeks. In the former group, 116 completed the full course of therapy; 2 patients died while on treatment (on day 93 and 151), 4 following the EOT (on day 41, 143, 166 and 328). The median follow-up of the surviving patients after EOT was 461 days (range 278-515 days).

All patients except 1 were Caucasian. Forty-seven percent of the grafts came from donors $\geq 65$ year old and/or with macrovesicular steatosis $\geq 15 \%$ (suboptimal graft) [13], $8 \%$ from hepatitis B core antibody positive donors and $7.1 \%$ from $\mathrm{HCV}$ antibody positive donorsenes.

Two patients had been treated with a first-generation HCV protease-inhibitor before LT. The baseline stage of hepatic fibrosis $(F)$ was evaluated in 28 patients by liver biopsy according to Metavir score (3 F3, 25 F4), in 12 patients by combined liver biopsy and transient elastography (12 F4) and in 86 patients by transient elastography only (27 F3 and 59 F4). The median LS was: $11 \mathrm{kPa}$ [interquartile 10.1-12] in the F3 group and 21.8 kPa [17-34.8] in the F4 group. Thirty-four patients had diabetes mellitus, 33 were insulin dependent at the beginning of antiviral therapy.

AllThe 8 patients treated with sofosbuvir, simeprevir and ribavirin were immunosuppressed with tacrolimus. The HCV genotype was $1 \mathrm{a}$ in two and $1 \mathrm{~b}$ in six. Seven patients had fibrosis Metavir F3 and 1 F4 (Child A5, MELD 7), with median HCV RNA $6.56 \log _{10} \mathrm{IU} / \mathrm{mL}$. 
Further demographic and baseline characteristics are presented in Table 1.

Four weeks after antiviral therapy was started, a statistically significant decrease was observed in the AST and ALT median values from 67 IU/L [45-114] to 24 IU/L [18-33], p $<0.001$, and $58 \mathrm{IU} / \mathrm{L}$ [40-100] to $18 \mathrm{IU} / \mathrm{L}$ [13-26], $\mathrm{p}<0.001$, respectively. Transaminase values normalized (Figure S1) and remained normal throughout the follow-up in all eradicated patients but nine, whose had persistently elevated enzymes transaminasespersisted slightly elevated-(less than twice upper limit of normal). Six of these latter patients underwent a liver biopsy which showed minimal histological changes without evidence of rejection. After 1 week of therapy, the median decrease in HCV RNA was 3.87 $\log _{10} \mathrm{H} / \mathrm{mL}$ in F3 and $3.55 \log _{10} \mathrm{HU} / \mathrm{mL}$ in F4 group $(p=0.002)$ (Figures 52 and S3). The overall SVR24 rate was $81.0 \%$ (102/126 patients, 95\% confidence interval 73.2-86.9) by intention to treat (ITT) analysis (Figure 1). The SVR24 rate was significantly higher in F3 patients-compared with F4 patients ones $(30 / 30,100 \%$ vs $72 / 96,75 \%, p=0.001)$; and in patients tolerating $\geq 80 \%$ of the full weight-based ribavirin dose $(n=104)$ compared with those tolerating $<80 \%$ of the dose $(n=22)(85.6 \%$ vs $59.0 \%, p=0.01)$ and in patients with preserved liver function (MELD $\leq 11$ and Child $\leq \mathrm{A} 6$, according to ROC curves) (Figure S2). Two cirrhotic patients who became HCV RNA negative on therapy, died for multiresistant bacteria-related septic shock, two others died of liver failure after the EOT: one, 68 years old, had achieved a sustained virological response at week 4 after EOT, the other, 44 years old, had achieved SVR12 and was on the list for retransplantation. Twenty patients experienced a relapsed of HCV (17 at week 4, 2 at week 12 and 1 at week 24 of follow-up; the latter cleared HCV RNA at week 4 on therapy and normalized transaminases at week 1 ). All relapsers were cirrhotic (9 patients Child A, 10 Child B and 1 Child C). They had a median pre-treatment MELD of 13 and had received sofosbuvir and ribavirin; a the majority of them were IL28B non-C/C genotype (80\%) and experienced to previous antiviral therapy (65\%) (Tables S1 and S2). Basal median LS was $28.0 \mathrm{kPa}$ in 
the relapsers and $20.4 \mathrm{kPa}$ in the cirrhotics who achieved viral eradication $(p=0.01)$. APRI and FIB-4 were significantly higher in relapsers compared with responders at baseline

\section{(3.27 vs 1.92 and 8.45 vs 5.95 , respectively; $p<0.01$ for both) (Table 2 ).}

From baseline to EOT, theif median Child improved in relapsers from B7 to A6 $(p=0.014)$, while the median MELD remained 13. Two patients died at 138 and 160 days after relapse due to progressive end-stage liver disease. Seventeen patients started a second treatment course, at a median of 17 weeks (range 5-43 weeks) from the relapse. No significant variation in liver function tests was observed between relapse and retreatment.(Table S2). One patient refused retreatment.

Iwo-cirrhotic patients who were HCV RNA negative on therapy, died for multiresistant bacteria-related septic shock; 2 others died of liver failure after the EOT: one, 68 years old, had achieved a sustained virological response at week 4 after EOT, and one, 44 years old, had achieved SVR12 and was on the list for retransplantation. In the cirrhotic patients, significant predictors of SVR24 at univariate analysis (Figure 2) were $M E L D \leq 11$, Child $\leq A 6$ and $L S \leq 26 \mathrm{kPa}$. The cut-offs were chosen according to ROC eurves: area under the curve (AUC) for MELD 0.723, $p=0.001$; Child $0.759, p<0.001$ and LS $0.751, p=0.002$ (Figures $S 4$ and S5).

Basal median LS was $28.0 \mathrm{kPa}$ in the relapsers and $20.4 \mathrm{kPa}$ in the cirrhotics whoachieved viral eradication $(p=0.01)$. APRI and FIB-4 at baseline were significantly higher in relapsers compared with responders ( 3.27 vs 1.92 and 8.45 vs 5.95 , respectively; $p<0.01$ for both) (Table S3).

Overall, in the cirrhotic group, between baseline and the last available follow-up, 20 patients $(20.8 \%)$ showed had a stable MELD from baseline to the last available follow-up

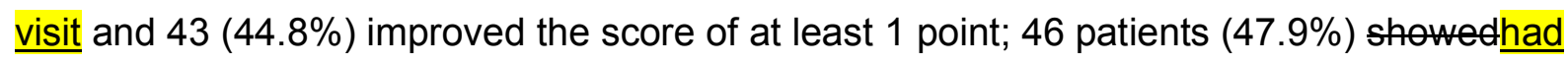
a stable Child and 35 (36.5\%) improved the score of at least 1 point. 


\section{Clinical outcomes in patients who achieved viral eradication}

In the F3 patients, liver and kidney function remained normal (Table S $\underline{3} 4$ ). APRI and FIB-4 were significantly lower at week 24 after EOT, compared with pre-treatment median values ( 1.07 to $0.41, p<0.001$, and 3.26 to $2.08, p<0.001$, respectively); these indexes remained stable in the 28 patients who reached 48 weeks of follow-up (Figure 13, Panels A and C). LS (available in 27 patients) improved from $11.0 \mathrm{kPa}$ [10.1-12.0] to $7.7 \mathrm{kPa}$ [6.6-10.2], $\mathrm{p}$ $<0.001$, at week 24 and remained stable at week 48 (7.8 kPa [7.0-10.6], $p=0.65$ vs week 24) (Figure 24, Panel A). In 18 patients (66.7\%) LS decreased below the $9.5 \mathrm{kPa}$ cut-off for F3 at week 48.

At week 24 after EOT, the 72 F4 patients who had achieved viral eradicationeradicated $\underline{\mathrm{HCV}}$, showedexhibited a significant improvement in platelets count, bilirubin and albumin levels, degree of ascites and Child score at pair wise comparison with baseline values [the rate of Child A was $77.8 \%$ (56/72) at baseline and $88.9 \%$ (64/72) at week 24 of follow-up]; kidney function and MELD score did not vary. Seventy-one of the 72 responders reached 48 weeks of follow-up and showed a further significant increase in platelets count; albumin, ascites, and Child score [93\% (66/71) Child A] remained stable (Table $\underline{\mathbf{3 2}}$ ).

Both APRI and FIB-4 decreased significantly at week 24 after EOT compared with baseline values (1.92 to $0.55, p<0.001$, and 5.95 to $3.15, p<0.001$, respectively), and further decreased at week 48, compared with 24 week values (APRI 0.51, p <0.001; FIB-4 3.12, p $<0.001)$ (Figure 13, Panels B and D). Fifty-one of the 72 patients underwent transient elastography at baseline and at SVR24; the median LS improved from $20.4 \mathrm{kPa}$ [16.033.8] to $17.5 \mathrm{kPa}$ [11.1-25.7], $\mathrm{p}<0.001$ (Figure 24, Panel B). Fifty patients underwent transient elastography also at week 48 after EOT; the median LS (14.0 kPa [10.0-21.3]) significantly decreased compared with week $24(p=0.001)$; in 21 of them $(42 \%)$ the LS fell below the $12.5 \mathrm{kPa}$ cut-off. 
As evaluated by transient elastography in F3-F4 patients pooled together ${ }_{2} \underline{\operatorname{In}}$ in $39 \underline{(50.6 \%)}$ of the $77(50.6 \%)$ F3-F4 responders pooled together, the fibrosis stage had regressed in

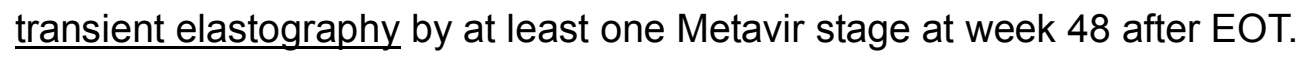

No patients developed hepatocellular carcinoma in the period of observation.

Thirteen out of the 20 cirrhotic responders with large oesophageal varices at baseline, underwent a second 48 week follow-up endoscopy; no change in the size of varices was observed. which showed nochanges.

\section{Safety}

AE are summarized in Table $\mathbf{S} \underline{45}$.

During therapy, seven cirrhotic patients were hospitalised: two patients for grade 3 encephalopathy, two for severe variceal bleeding and three for sepsis in decompensated liver disease. Six cirrhotic patients died of complications of end-stage liver disease, unrelated to antiviral therapy.

Three patients discontinued ribavirin for less than 1 week due to diffuse macular skin rash and no one discontinued therapy permanently due to $A E$.

Because of anemia and/or compromised renal function, $34.1 \%(43 / 126)$ of the patients were treated with a non weight-based escalating dose of ribavirin, starting from $400 \mathrm{mg}$ daily and $11.1 \%(14 / 126)$ required dosage reduction; however, $82.5 \%(104 / 126)$ received at least $80 \%$ of the weight-based dosage. Median daily ribavirin dose was $1000 \mathrm{mg}$ from baseline to EOT and median haemoglobin level declined from $12.8 \mathrm{~g} / \mathrm{dL}$ at baseline to 10.9 $\mathrm{g} / \mathrm{dL}$ at week 4 and remained stable around $10.8 \mathrm{~g} / \mathrm{dL}$ from week 8 to week 24 (Figure S부). In 60 patients (47.6\%) we measured at least one haemoglobin level of less than 10 $\mathrm{g} / \mathrm{dL}$ and in $12(9.5 \%)$ of less than $8.5 \mathrm{~g} / \mathrm{dL} ; 45$ patients (35.7\%) received epoetin and 14 $(11.1 \%)$ required blood transfusion. 
Minimal dose adjustments in the calcineurin inhibitors were required throughout the treatment in $64 \%$ of the patients $(27 \%$ increased, $12 \%$ decreased and $25 \%$ both decreased and increased the dose) to maintain stable trough levels. No biopsy-proven acute rejection occurred.

\section{DISCUSSION}

The advent of highly effective and well tolerated DAAs against the HCV is revolutionizing the outcome of LT for hepatitis C. In this setting, early treatment after surgery is expected to be the most cost-effective strategy for patients who are still viremic at LT $[10,14]$, nevertheless DAAs are providing rescue also to residual cohorts of re-infected transplant recipients who developed advanced HCV disease $[7,8,15]$. When sofosbuvir became available in Italy in June 2014 , these patients received priority access to the new therapy through a compassionate use program. A majority were treated with sofosbuvir and ribavirin; a small minority received the combination of sofosbuvir, ribavirin and simeprevir, which became available almost at the end of the enrollment period.

After a median follow-up of 461 days from EOT, $102(81 \%)$ of our 126 patients are free of HCV and clinically stable. SVR was obtained also in a high proportion (95\%) of the 21 patients with genotype 3 , though this genotype is the most difficult to treat in the ordinary clinical context. All treatment failures, excluding deceased patients, were due to virologic relapses and all relapses, except one, occurred within the first 12 weeks after EOT. Response to therapy was independent from gender, HCV genotype, basal viremia, rapidvirologic response, HLA DRB1*11 phenotype and IL28B polymorphisms, while it was influenced by the stage of fibrosis and by intolerance to ribavirin: $75 \%$ of the patients with cirrhosis achieved an SVR compared to $100 \%$ of those with F3 fibrosis and among the 22 patients who tolerated less than $80 \%$ of the optimal ribavirin dosage, only $59 \%$ obtained an SVR. As expected, anemia was the main complication of ribavirin. 
Considering the higher proportion of patients with cirrhosis in this study, our results appear better than the $70 \%$ rate of SVR12 reported by Charlton [16] in a smaller series of 40 patients recruited in a multicenter study, of whom only $40 \%$ were cirrhotics; possibly the management of the patients in a single center adopting a common approach may lead to more uniform results. Though our results are distinctly better than the $43 \%$ response reported by Forns et al [7], their series included a mixed population of compensated and decompensated cirrhotics in which a substantial number of patients had a higher MELD score and more severe disease than our patients.

There were in our study six deaths due to complications of the liver disease, two occurring in patients who had cleared the HCV. The deceased patients had a higher MELD score, ranging from 14 to 19 , in confirmation that a deteriorated liver function may prevent clinical improvement despite the antiviral efficacy of treatment [17]; vice versa a preserved liver function expressed by a Child Pugh score $\leq 6$ and a MELD $\leq 11$ was associated with SVR. Unfortunately, the threshold that may predict the lack of a clinical advantage despite the efficacy of therapy is difficult to establish [17].

In our 72 cirrhotic responders, SVR24 was associated with the normalization of aminotransferases in almost all patients, accompanied by a decrease of bilirubin and an increase of albumin levels; renal function deteriorated in none and ascites disappeared in three quarters of cases. The platelet count increased throughout therapy, presumably related to the administration of epoetin and to an increased production of endogenous erythropoietin, secondary to ribavirin-induced anemia [18]. The platelet number diminished to baseline values at week 12 post-therapy, but then climbed back significantly at week 24 and further increased at week 48; this trend and the regression of ascites may might be expression of an initial reduction in portal hypertension.

The APRI and FIB-4 indexes significantly improved throughout the follow-up; as aminotransferases and platelets are major components for their calculation, the 
improvement can be explained by the normalization of the enzymes and by the increase in the platelet count.

Transient elastography LS values were significantly reduced at the $6^{\text {th }}$ month of follow-up; this early improvement presumably resulted for a large extent byfrom the decrease of inflammation and edema which may artificially alter elastography. Interestingly, however, LS values showed a further significant decrease during the subsequent 6 month follow-up in patients with cirrhosis. Considering that artefacts due to liver inflammation could no longer be implicated, it is plausible that the observed continuing decrease of LS after the SVR24 corresponded to a genuine progressive reduction of fibrosis. In agreement with previous findings of fibrosis reversion in non-transplant patients with HCV cirrhosis who responded to interferon-therapy [19-21], our data would suggest that regression of fibrosis starts early and may become already measureable by transient elastography in the first year after therapy.

In conclusion, our experience in a large single center LT cohort of advanced recurrent HCV disease, confirms the major therapeutic impact of sofosbuvir with ribavirin, extending also to difficult-to-treat genotype 3 patients.

We acknowledge that a new wave of more efficacious DAA combinations is now available to treat hepatitis $C[8,15,22]$. Nevertheless, this study provides a paradigm of the excellent clinical outcome and of the potential for disease reversibility after that can beexpected with-successful DAA therapy in the transplant setting.

\section{ACKNOWLEDGEMENTS}

No specific funding was obtained for this study. 


\section{REFERENCES}

[1] Adam R, Karam V, Delvart V. Evolution of indications and results of liver transplantation in Europe. A report from the European Liver Transplant Registry (ELTR). J Hepatol 2012;57:675-688.

[2] Forman LM, Lewis JD, Berlin JA, Feldman HI, Lucey MR. The association between hepatitis $\mathrm{C}$ infection and survival after orthotopic liver transplantation. Gastroenterology 2002;122:889-896.

[3] Crespo G, Marino Z, Navasa M, Forns X. Viral hepatitis in liver transplantation. Gastroenterology 2012;142:1373-1383.

[4] Berenguer M. Systematic review of the treatment of established recurrent hepatitis C with pegylated interferon in combination with ribavirin. J Hepatol 2008;49:274-287.

[5] Coilly A, Roche B, Dumortier J, Leroy V, Botta-Fridlund D, Radenne S, et al. Safety and efficacy of protease inhibitors to treat hepatitis $\mathrm{C}$ after liver transplantation: a multicenter experience. J Hepatol 2014;60:78-86.

[6] EASL recommendations on treatment of hepatitis C 2015. J Hepatol 2015;63:199236.

[7] Forns X, Charlton M, Denning J, McHutchison JG, Symonds WT, Brainard D, et al. Sofosbuvir compassionate use program for patients with severe recurrent hepatitis $C$ after liver transplantation. Hepatology 2015;61:1485-1494.

[8] Brown RS Jr, O'Leary JG, Reddy KR, Kuo A, Morelli GJ, Burton JR Jr, et al. Interferon-free therapy for genotype 1 hepatitis $C$ in liver transplant recipients: real world experience from HCV-TARGET. Liver Transpl 2016;22:24-33.

[9] Castera L, Forns X, Alberti A. Non-invasive evaluation of liver fibrosis using transient elastography. J Hepatol 2008;48:835-847.

[10] Romagnoli R, Martini S, Tandoi F, Dell Olio D, Magistroni P, Bertinetto FE, et al. Early reduced liver graft survival in hepatitis $C$ recipients identified by two combined 
genetic markers. Transpl Int 2016; doi: 10.1111/tri.12795.

[11] Vallet-Pichard A, Mallet V, Nalpas B, Verkarre V, Nalpas A, Dhalluin-Venier V, et al. FIB-4: an inexpensive and accurate marker of fibrosis in HCV infection. Comparison with liver biopsy and fibrotest. Hepatology 2007;46:32-36.

[12] Wai CT, Greenson JK, Fontana RJ, Kalbfleisch JD, Marrero JA, Conjeevaram HS, et al. A simple non-invasive index can predict both significant fibrosis and cirrhosis in patients with chronic hepatitis C. Hepatology 2003;38:518-526.

[13] Salizzoni M, Franchello A, Smedile A, David E, Barbui A, Torrani M, et al. Marginal grafts: finding the correct treatment for fatty livers. Transpl Int 2003;16:486-493.

[14] Russo FP, Zanetto A, Burra P. Timing for treatment of HCV recurrence after liver transplantation: the earlier the better. Transpl Int 2015;2016;29:694-697. doi: $10.1111 /$ tri. 12739 .

[15] Charlton M, Everson GT, Flamm SL, Kumar P, Landis C, Brown RS, et al. Ledipasvir and sofosbuvir plus ribavirin for treatment of HCV infection in patients with advanced liver disease. Gastroenterology 2015;149:649-659.

[16] Charlton M, Gane E, Manns MP, Brown RS Jr, Curry MP, Kwo PY, et al. Sofosbuvir and ribavirin for treatment of compensated recurrent hepatitis $\mathrm{C}$ virus infection after liver transplantation. Gastroenterology 2015;148:108-117.

[17] Pellicelli AM, Montalbano M, Lionetti R, Durand C, Ferenci P, D’Offizi G, et al. Sofosbuvir plus daclatasvir for post-transplant recurrent hepatitis C: potent antiviral activity but no clinical benefit if treatment is given late. Dig Liver Dis 2014;46:923-927.

[18] Kobayashi T, Hige S, Terashita K, Nakai M, Horimoto H, Sho T, et al. Anemia and thrombocytosis induced by ribavirin monotherapy in patients with chronic hepatitis $\mathrm{C}$. J Gastroenterol 2012;47:1228-1237.

[19] Poynard T, McHutchison J, Manns M, Trepo C, Lindsay K, Goodman Z, et al. Impact of pegylated interferon alfa-2b and ribavirin on liver fibrosis in patients with chronic 
hepatitis C. Gastroenterology 2002;122:1303-1313.

[20] EASL-ALEH clinical practice guidelines: non-invasive tests for evaluation of liver disease severity and prognosis. European Association for the Study of the Liver. J Hepatol 2015;63:237-264.

[21] D'Ambrosio R, Aghemo A, Fraquelli M, Rumi MG, Donato MF, Paradis V, et al. The diagnostic accuracy of Fibroscan ${ }^{\circledR}$ for cirrhosis is influenced by liver morphometry in HCV patients with a sustained virological response. J Hepatol 2013;59:251-256.

[22] Foster GR, Irving WL, Cheung MC, Walker AJ, Hudson BE, Verma S, et al. HCV

Research UK. Cohort study of the impacts of direct acting antiviral therapy in patients with chronic hepatitis C and decompensated cirrhosis. J Hepatol 2016;64:1224-1231. 
Table 1. Demographic and baseline characteristics of recipients and donors

\begin{tabular}{|c|c|}
\hline \multicolumn{2}{|l|}{ Recipient features $(N=126)$} \\
\hline Age (years) & $60[54-66]$ \\
\hline Male & $99(78.6 \%)$ \\
\hline Body Mass Index $\left(\mathrm{Kg} / \mathrm{m}^{2}\right)$ & $24.6[22.5-26.9]$ \\
\hline $25-\mathrm{OH}$ vitamin $\mathrm{D}^{\wedge}(<20 \mathrm{ng} / \mathrm{mL})$ & $71(59.2 \%)$ \\
\hline HLA-DRB1*11 positive phenotype & $34(27.0 \%)$ \\
\hline \multicolumn{2}{|l|}{ IL28B genotype } \\
\hline rs $12979860 \mathrm{C} / \mathrm{C}$ & $32(25.4 \%)$ \\
\hline rs $8099917 \mathrm{~T} / \mathrm{T}$ & $59(46.8 \%)$ \\
\hline Previous antiviral treatment & $83(65.9 \%)$ \\
\hline Antiviral treatment after LT & $57(45.2 \%)$ \\
\hline \multicolumn{2}{|l|}{ Immunosuppressive drug } \\
\hline CsA - Tac - MMF - other & $50(39.7 \%)-56(44.4 \%)-56(44.4 \%)-5(4.0 \%)$ \\
\hline Time since LT (years) & $4.3[2.3-7.2]$ \\
\hline \multicolumn{2}{|l|}{ HCV genotype } \\
\hline $1 a-1 b-1$ (unspecified) & $17(13.5 \%)-70(55.6 \%)-6(4.8 \%)$ \\
\hline $2-3-4$ & $6(4.8 \%)-21(16.7 \%)-6(4.8 \%)$ \\
\hline Baseline HCV RNA $\left(\log _{10} \mathrm{IU} / \mathrm{mL}\right)$ & $6.40[6.10-6.66]$ \\
\hline$\geq 10^{6} \mathrm{IU} / \mathrm{mL}$ & $101(80.2 \%)$ \\
\hline
\end{tabular}

\section{Corresponding Metavir fibrosis stage}
F3 - F4
$30(23.8 \%)-96(76.2 \%)$

\begin{tabular}{lc}
\hline Haemoglobin $(\mathrm{g} / \mathrm{dL})$ & $12.8[11.4-14.4]$ \\
\hline Platelets count $\left(10^{9} / \mathrm{L}\right)$ & $99[67-140]$ \\
\hline Liver enzymes $(\mathrm{IU} / \mathrm{L})$ & $60[40-101]$ \\
Aspartate aminotransferase & $69[45-116]$ \\
Alanine aminotransferase & $75[41-152]$ \\
Y-Glutamyltransferase &
\end{tabular}


Numerical variables are expressed as median [Q1-Q3]. Categorical variables are expressed as numbers (prevalence, \%).

${ }^{\wedge} 6$ missing data

${ }^{*}$ Chronic Kidney Disease Epidemiology Collaboration equation

$\S$ In 96 cirrhotic patients

\#According to Salizzoni et al, Transpl Int 2003

Abbreviations: CsA, cyclosporine; D-MELD, donor age x recipient MELD; eGFR, estimated glomerular filtration rate; F, fibrosis; $\mathrm{HCV}$, hepatitis $C$ virus; HLA, human leukocyte antigen; IL28B, Interleukin 28B; LT, liver transplantation; MELD, Model for End-stage Liver Disease; MMF, mycophenolate mofetil; Tac, tacrolimus. 
Table 2. Liver function tests and non-invasive markers of fibrosis at baseline and at the end of therapy in responder versus relapser cirrhotic patients

\begin{tabular}{|c|c|c|c|}
\hline BASELINE & $\begin{array}{l}\text { Responders } \\
\qquad(n=72)\end{array}$ & $\begin{array}{c}\text { Relapsers } \\
\qquad(n=20)\end{array}$ & $\mathbf{p}^{\#}$ \\
\hline APRI & $1.92[1.00-3.27]$ & $3.27[1.72-5.51]$ & 0.007 \\
\hline FIB-4 & $5.95[3.60-11.10]$ & $8.45[7.17-16.08]$ & 0.009 \\
\hline Liver stiffness* $(\mathrm{kPa})$ & $20.4[16.0-33.8]$ & 28.0 [21.6-44.4] & 0.01 \\
\hline Child score & $5[5-6]$ & 7 [6-8] & 0.002 \\
\hline MELD score & 10 [8-12] & 13 [9-16] & 0.02 \\
\hline EOT & $\begin{array}{c}\text { Responders } \\
\quad(n=72)\end{array}$ & $\begin{array}{l}\text { Relapsers } \\
\qquad(n=20)\end{array}$ & $\mathbf{p}^{\#}$ \\
\hline APRI & $0.46[0.29-0.76]$ & $0.83[0.57-1.22]$ & 0.02 \\
\hline FIB-4 & $3.25[1.90-4.30]$ & $4.80[3.71-7.42]$ & 0.01 \\
\hline Liver stiffness* $(\mathrm{kPa})$ & $17.5[11.1-25.7]$ & 23.4 [18.3-39.0] & 0.09 \\
\hline Child score & $5[5-6]$ & $6[5-7]$ & 0.19 \\
\hline MELD score & $11[9-14]$ & $13[11-17]$ & 0.05 \\
\hline
\end{tabular}

Numerical variables are expressed as median [Q1-Q3]

*Liver stiffness was available at baseline in 51 responders and 17 relapsers; at EOT in 51 responders and 13 relapsers

" p-values calculated according to Wilcoxon test for paired data

Abbreviations: APRI, AST to platelet ratio index; EOT, end of therapy; kPa, kilopascal; MELD, Model for Endstage Liver Disease. 
Table $\underline{32}$. Clinical and biochemical variations in cirrhotic long-term responder cirrhotic patients-who achieved viral eradication

\begin{tabular}{|c|c|c|c|c|c|c|c|}
\hline & $\begin{array}{l}\text { Baseline } \\
(\mathrm{N}=72)\end{array}$ & $\begin{array}{c}\text { EOT } \\
(\mathrm{N}=72)\end{array}$ & $\begin{array}{l}\text { SVR12 } \\
(N=72)\end{array}$ & $\begin{array}{l}\text { SVR24 } \\
(N=72)\end{array}$ & $\begin{array}{l}\text { SVR48 } \\
\left(\mathrm{N}=71^{\circ}\right)\end{array}$ & $p^{\wedge}$ & $p^{\S}$ \\
\hline AST (IU/L) & 69 [47-97] & $23[20-28]$ & 27 [22-35] & 24 [18-32] & 21 [18-28] & $<0.001$ & 0.13 \\
\hline ALT (IU/L) & 59 [41-90] & 15 [11-23] & $18[14-27]$ & 16 [12-25] & $17[12-27]$ & $<0.001$ & 0.02 \\
\hline Bilirubin (mg/dL) & $1.1[0.8-1.5]$ & $1.4[1.1-2.2]$ & $0.9[0.6-1.2]$ & $0.8[0.6-1.2]$ & $0.9[0.6-1.1]$ & $<0.001$ & 0.74 \\
\hline INR & $\begin{array}{c}1.06 \\
{[0.99-1.20]}\end{array}$ & $\begin{array}{c}1.11 \\
{[1.07-1.24]}\end{array}$ & $\begin{array}{c}1.09 \\
{[1.02-1.24]}\end{array}$ & $\begin{array}{c}1.08 \\
{[1.02-1.22]}\end{array}$ & $\begin{array}{c}1.11 \\
{[1.07-1.23]}\end{array}$ & 0.03 & 0.007 \\
\hline Albumin (g/dL) & $3.9[3.6-4.3]$ & $4.1[3.8-4.4]$ & $4.1[3.7-4.3]$ & $4.2[3.8-4.4]$ & $4.3[4.0-4.5]$ & 0.02 & 0.31 \\
\hline Creatinine (mg/dL) & $1.1[0.9-1.3]$ & $1.1[0.9-1.3]$ & $1.0[0.9-1.3]$ & $1.1[1.0-1.3]$ & $1.1[0.9-1.3]$ & 0.36 & 0.12 \\
\hline eGFR* (mL/min) & 68 [52-84] & $64[52-80]$ & 67 [53-83] & $63[52-78]$ & $62[52-82]$ & 0.26 & 0.25 \\
\hline Platelets $\left(10^{9} / \mathrm{L}\right)$ & 99 [65-134] & 131 [86-185] & 99 [64-133] & $108[72-142]$ & 112 [68-144] & 0.002 & 0.01 \\
\hline Ascites & $22(30.6 \%)$ & $12(16.7 \%)$ & $8(11.1 \%)$ & $7(9.7 \%)$ & $7(9.9 \%)$ & 0.003 & $>0.99$ \\
\hline Encephalopathy & $5(6.9 \%)$ & $4(5.6 \%)$ & $3(4.2 \%)$ & $3(4.2 \%)$ & $2(2.8 \%)$ & 0.72 & $>0.99$ \\
\hline MELD score & $10[8-12]$ & $11[9-14]$ & 9 [8-11] & 9 [8-11] & 10 [8-11] & 0.34 & 0.92 \\
\hline Child score & $5[5-6]$ & $5[5-6]$ & $5[5-6]$ & $5[5-5]$ & $5[5-5]$ & $<0.001$ & 0.68 \\
\hline
\end{tabular}

Numerical variables are expressed as median [Q1-Q3]. Categorical variables are expressed as numbers (prevalence,\%).

p-values calculated according to Wilcoxon test for paired data for numerical variables and according to Fisher's exact test for categorical variables.

${ }^{\circ} 71$ out of 72 cured patients reached 48 weeks of follow-up after therapy

${ }^{*}$ Chronic Kidney Disease Epidemiology Collaboration equation

^Baseline versus SVR24

§SVR24 versus SVR48

Abbreviations: ALT, alanine aminotransferase; AST, aspartate aminotransferase; eGFR, estimated glomerular filtration rate; EOT, end of therapy; MELD, Model for End-stage Liver Disease; SVR12, sustained virological response at week 12 after end of therapy; SVR24, sustained virological response at week 24 after end of therapy; SVR48, sustained virological response at week 48 after end of therapy. 


\section{FIGURE LEGENDS}

Figure 1. Sustained virological response rates by intention to treat analysisaccording to fibrosis stage. SVR, sustained virological response; F, fibrosis according to Metavir; SVR12, sustained virological response at week 12 after therapy; SVR24, sustained virological response at week 24 after therapy.

Figure 2. Sustained virological response rates at week 24 after therapy in the 96 cirrhotic patients. SVR24, sustained virological response at week 24 after therapy; $\mathrm{Cl}$, confidence interval; IL28B, Interleukin 28B; HLA, human leukocyte antigen; TW4, week 4 on therapy; MELD, Model for End-stage Liver Disease; APRI, AST to platelet ratio index; $\mathrm{kPa}$, kilopascal.

Figure 13. Variations of from baseline to weeks 24 and 48 after therapy in APRI (Panels A and B) and FIB-4 (Panels C and D) from baseline to weeks 24 and 48 after therapy, in F3 and F4 long-term responders. patients who achieved viral eradication. APRI, AST to platelet ratio index; F, fibrosis according to Metavir; SVR24, sustained virological response at week 24 after therapy; SVR48, sustained virological response at week 48 after therapy.

Figure 24. Variations inof liver stiffness from baseline to weeks 24 and 48 after therapy in F3 (Panel A) and F4 patients (Panel B) who-achieved viraleradicationlong-term responders. F, fibrosis according to Metavir; kPa, kilopascal; SVR24, sustained virological response at week 24 after therapy; SVR48, sustained virological response at week 48 after therapy. 
Figure 1. Variations of APRI (Panels A and B) and FIB-4 (Panels C and D) from baseline to weeks 24 and 48 after therapy, in F3 and F4 long-term responders. APRI, AST to platelet ratio index; F, fibrosis according to Metavir; SVR24, sustained virological response at week 24 after therapy; SVR48, sustained virological response at week 48 after therapy. $194 \times 236 \mathrm{~mm}(72 \times 72 \mathrm{DPI})$
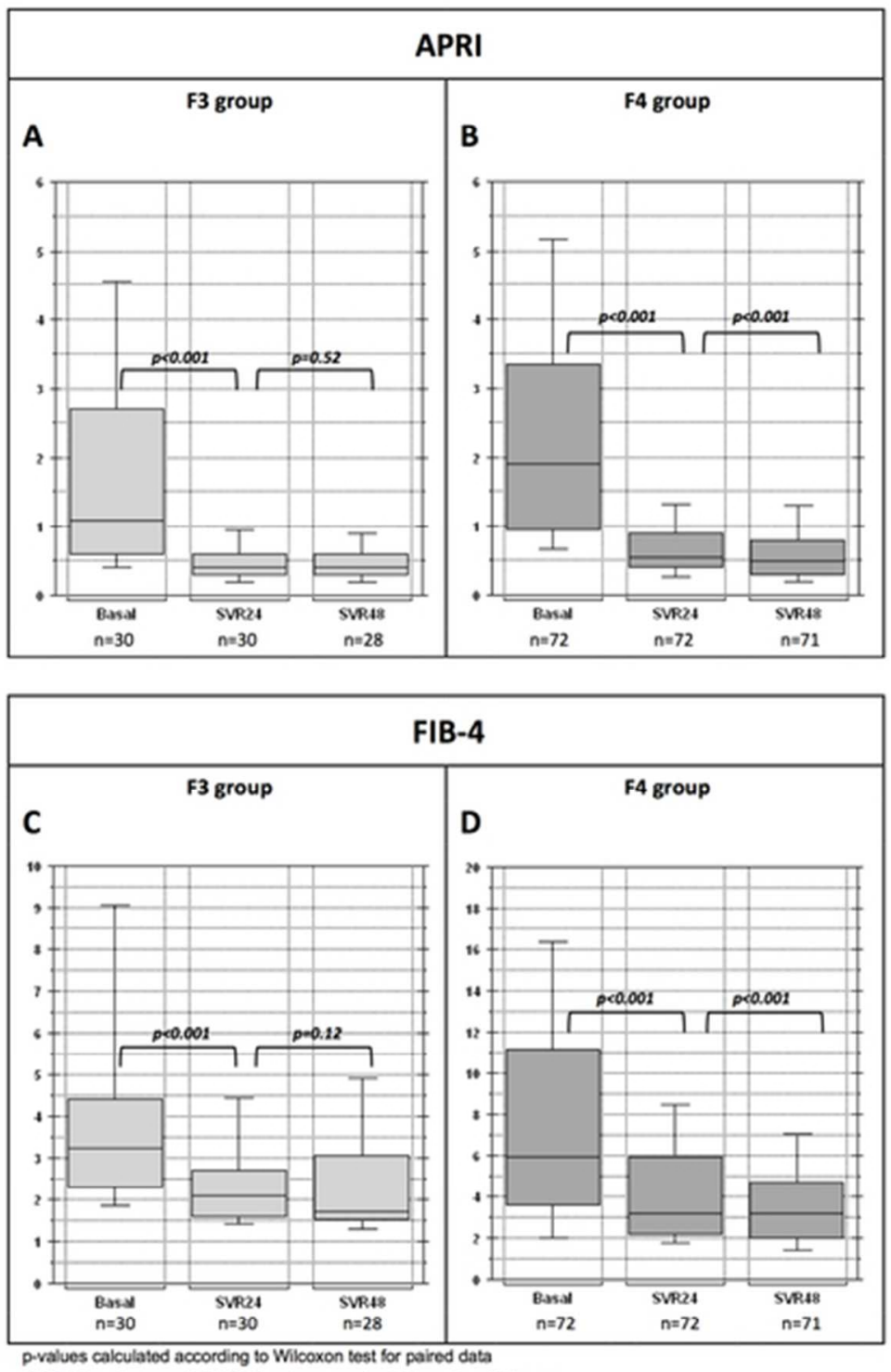

$28 / 30 \mathrm{~F} 3$ patients and $71 / 72 \mathrm{~F} 4$ patients reached 48 weeks of follow-up. 
Figure 2. Variations of liver stiffness from baseline to weeks 24 and 48 after therapy in F3 (Panel A) and F4 (Panel B) long-term responders. F, fibrosis according to Metavir; kPa, kilopascal; SVR24, sustained virological response at week 24 after therapy; SVR48, sustained virological response at week 48 after therapy. 